\title{
Validation of Corrupted Medical Image Labelling using Deep Neural Network
}

Mhd Khaled Maen ${ }^{1}$, Amelia Ritahani Ismail ${ }^{1}$, M.A.B. M.D. Ali ${ }^{2}$, Nadzurah Zainal Abidin ${ }^{1}$

${ }^{1}$ Department of Computer Science, Kulliyyah of Information and Communication Technology, International Islamic University,Gombak Selangor

${ }^{2}$ Department of Physics, Kulliyyah of Science, International Islamic University Malaysia Kuantan Pahang.

Introduction: Deep Neural Networks (DNN) has been successfully applied in a variety of medical image analysis. However, in building a good DNN model, it requires validated label from medical experts. The task of getting labels validated by the medical expert is quite a challenge due to the complexity of the image as well as large variability between the experts. Thus, this research investigates the effect of corrupted medical label on deep neural network for analysing diseases. Materials and method: In investigating this issue, a chest X-ray dataset comprising of 2560 images with 14 multilabel diseases from the National Institutes of Health (NIH) are examined. The DNN models that are explored are MobileNet, which is a base network that uses a depth-wise separable convolutional in building lightweight DNN and Xception, which is also utilised depth-wise separable convolutional with inception modules as an intermediate step in between regular convolutional and the depthwise convolutional. Different training and testing sizes are presented to the models with different ranges of corrupted labels from 10\% to 50\%. The training and validation accuracy is compared for each of the test cases together with other statistical analysis. Results: Based on the conducted experiments, it can be highlighted that both models can classify the diseases with the accuracy of more than $80 \%$ if the images have been previously trained by the model for different sizes of the corrupted labels. For the unseen or previously untrained images, the models can reach the accuracy of $70 \%$ and as the sizes of corrupted label increases, the accuracy will decreases as low as $50 \%$. Conclusion: To conclude, corrupted labels have a critical effect on the DNN models. Thus, it is very essential to study the effect of the corrupted medical images label to ensure the reliability of the DNN models for medical imaging. 